Brief communication

\title{
Perception of Vulnerability and Ruminant Thoughts about COVID-19 in Spanish Students
}

\author{
José-María Figueredo $^{1 *}$, Cristina García-Ael ${ }^{2}$ and Gabriela Topa ${ }^{2}$ \\ 1 UNED, International Doctorate School; ifiguered4@alumno.uned.es (J.M.F.) \\ 2 National Distance Education University UNED; cgarciaael@psi.uned.es (C.G.A.); gtopa@psi.uned.es (G.T.) \\ * Correspondence: José-María Figueredo, ; jfiguered4@alumno.uned.es (tel: +34 661308065)
}

Publisher's Note: MDPI stays neutral with regard to jurisdictional claims in published maps and institutional affiliations.

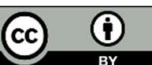

Copyright: $\odot 2021$ by the authors. Submitted for possible open access publication under the terms and conditions of the Creative Commons Attribution (CC BY) license (http://creativecommons.org/licenses/by/4.0/).

\begin{abstract}
The current situation in schools in relation to COVID-19 can generate a decrease in academic performance due to factors intrinsic to students. Therefore, rumination about COVID-19 could interfere with students' attention, resulting in a decrease in their academic performance. Therefore, the objective of this study was to explore the relationship between the perception of vulnerability to the disease and rumination about COVID-19 from a cross-sectional sample of postcompulsory education students. Our data suggest a positive relationship between the perception of vulnerability to the disease and ruminants' thoughts about COVID-19 $(r=.23)$
\end{abstract}

Keywords: COVID-19; rumination; academic performance; perceived vulnerability to disease

\section{Introduction}

At the beginning of 2020, the disease caused by the coronavirus (COVID-19) broke into our lives. The continuous news about COVID-19, the global economic crisis and the measures adopted by the governments of the different countries to stop the pandemic have resulted in an increase in feelings of anxiety and depression in the general population [1]. These feelings of anxiety and depression cause an increase in intrusive or ruminant thoughts [2]. Ruminant thoughts are intrusive, repetitive, passive and automatic thoughts about negative personal concerns and about the possible implications of a negative state [3]. On the basis of this literature, we propose the hypothesis (H1): the level of ruminant thoughts about COVID-19 will be medium or high among post-compulsory education students.

The tendency to have ruminant thoughts depends on individual differences in the cognitive processes that control information processing [4]. Thus, rumination will be associated with a decrease in the ability to inhibit information that is not relevant and distracting [5-6], and with other non-inhibitory forms of control [7], such as excessive autofocus [8]. However, the level of rumination may be associated with other individual traits [8]. On the other hand, it has been confirmed that the fear of being infected by COVID-19 is positively related to depression, anxiety and the perception of vulnerability to the disease [9]. The Perception of Vulnerability to Disease can be defined as personal beliefs about the susceptibility of contracting contagious diseases and the emotional distress associated with this possible contagion [10]. The perception of vulnerability to the disease is positively related to disgust [11] and with the rejection of people who pose a threat of infection [12]. Based on these findings, and taking into account that the relationships between perception of vulnerability to the disease and ruminant thinking about COVID-19 has not been examined, we propose our second hypothesis (H2): the perception of vulnerability to the disease will be directly and positively associated with the level of rumination on COVID-19.

Examining the personal factors that trigger rumination in academic contexts seems to us to be a particularly relevant topic for several reasons. Ruminant thoughts cause a 
variety of negative symptoms [13]. For example, ruminant thoughts are positively associated with stress [14], procrastination in students [15], and burnout at work [16], and negatively with motivation and concentration [17], and with vigor at work [16]. Ruminant thoughts continuously consume cognitive and energy resources, causing interference in attention [18], concentration and motivation [17] and as a consequence, non-task-related rumination it can cause a decrease in the individual's performance in academic tasks [19]

\section{Method}

\subsection{Participants}

The sample consisted of 109 post-compulsory education students ( $\mathrm{n}$ women $=97$; $89 \%)$, all of them older than 18 years, with a mean age of 23.29 years $(\mathrm{SD}=6.88)$. At the time of the research, 75 subjects $(68.8 \%)$ were studying vocational training, $30(27.5 \%)$ university degree, $2(1.8 \%)$ master's degrees and 2 doctoral studies $(1,8 \%)$. $28.4 \%$ of the subjects combined their studies with work, and $11.9 \%$ were looking for a job. Regarding the level of studies, the majority had high school studies $(n=54 ; 49.5 \%)$ or professional training $(n=36 ; 33 \%)$, the rest being university graduates $(n=19 ; 17.5) .13 .8 \%$ of the subjects reported having a chronic or long-term illness.

\subsection{Instruments}

Participants rated their responses on a five-point scale $(1=$ strongly disagree, $5=$ strongly agree).

Rumination about COVID-19. It was measured with a modified version of the rumination subscale of the cognitive regulation of emotions questionnaire [20]. We follow the procedure followed by Bakker \& van Wingerden [16]. In this way, each item on the scale made specific reference to COVID-19, for example "This week, I was often thinking about how I feel about the coronavirus". Cronbach's alpha coefficient was 0.91 .

Perception of vulnerability to disease. It was measured with the Perceived Vulnerability to Disease Scale (PVD) [20], validated in Spanish by Magallares et al. [10]. PVD is made up of two factors: perceived infectivity and aversion to germs. The perceived infectivity subscale refers to personal perception of the risk of being infected, while the germ aversion subscale examines rejection or fear of germs. The reliability of the general scale was acceptable $(\propto=0.7)$

\subsection{Process}

The sample was obtained by non-probability sampling. The participants were informed of the objectives of the study and the anonymity of the collected data. Participation was voluntary, and the participants in the final sample gave their consent to participate in the study and could withdraw from the study whenever they wanted. After expressing their consent to participate in the research, the participants individually filled in the questionnaire scales (Rumiantion, PVD). The format of the questionnaires was online.

\subsection{Analysis of data}

Descriptive analyzes and correlations were carried out. Subsequently, a linear regression analysis was carried out between the variables rumination on covid and perception of vulnerability to the disease.

\section{Results}

\subsection{Descriptive and correlation analysis}

First, a bivariate correlation analysis was performed to find out the relationships between the variables. In all cases the assumptions of independence, collinearity and homoscedasticity were met (IVF was less than 5 and the tolerance index was greater than 0.2 in all cases, so our data verified the assumption of non-collinearity between the variables). 
As can be seen in Table 1, rumination on COVID-19 was directly and positively associated both with the perception of vulnerability to the disease $(r=.23)$ and with the perceived infectivity $(\mathrm{r}=.14)$ and the aversion to germs $(\mathrm{r}=.21)$

Table 1. Descriptive statistics and correlations.

\begin{tabular}{lcccccc}
\hline \multicolumn{1}{c}{ Variables } & $\boldsymbol{M}$ & SD & $\mathbf{1}$ & $\mathbf{2}$ & $\mathbf{3}$ & $\mathbf{4}$ \\
\hline 1. Rumination & 3.14 & 1.13 & 1 & & & \\
2. Infectibility & 2.80 & .71 & .14 & 1 & & \\
3. Aversion & 3.57 & .74 & $.21^{*}$ & $.21^{*}$ & 1 & \\
4. Perception of ulnerability & 3.19 & .56 & $.23^{*}$ & $.77^{* *}$ & $.78^{* *}$ & 1 \\
\hline${ }^{*} \mathrm{p}<.05{ }^{*} \mathrm{p}<.01 *^{* *}$. & & & & & &
\end{tabular}
${ }^{*} \mathrm{p}<.05 ;{ }^{*} \mathrm{p}<.01 ;{ }^{* *}$.

\subsection{Linear regression}

In order to delve into the relationships between rumination on COVID-19 and perception of vulnerability to the disease, we calculated a regression analysis between the variables. The results confirm that the level of rumination on COVID-19 can be predicted through the level of susceptibility to the disease through the equation $(\mathrm{y}=1.67+.46 \mathrm{X})$. Confidence intervals were estimated using the bootstrapping technique with 5000 subsamples $(\beta=.46, \mathrm{p}<.05 ; 95 \%$ CI [. 07.83$])$.

\section{Discussion}

The general objective of our research was to explore the relationships between rumination about COVID-19 and the perception of vulnerability to the disease. Our results confirm that the degree of ruminant thinking about COVID-19 in the student population is medium-high. Likewise, it is confirmed that there is a direct and positive relationship between ruminant thoughts about COVID-19 and perception of vulnerability to the disease. Our findings are consistent with some research examining certain negative psychological reactions that affect people's well-being during times of infectious epidemic crisis [21]

\subsection{Limitations}

Our research has certain limitations. The first limitation would be related to the use of self-report measures. The second limitation would be related to the sample. Our sample was made up of post-compulsory education students. In this sense, it would be important to examine different groups of students, of different educational levels. The final limitation is the cross-sectional nature of the research design. The hypothesized casual relationships should be confirmed in future longitudinal studies. Despite these limitations, our findings open a series of relevant avenues for future research.

\subsection{Future research}

Future research should examine the impact of ruminant thoughts about COVID-19 on student academic performance. Likewise, it would be necessary to deepen the knowledge about gender differences in terms of ruminant thoughts and the perception of vulnerability. Regarding academic performance, it would be necessary to measure the proportion of the variance that is due to the interference caused by ruminant thoughts about COVID-19. For this, it would be necessary to control other variables that currently have broken into teaching contexts (new methodologies that imply less presence), less direct supervision by teachers ... as well as the classic variables (study time, perceived selfefficacy, commitment in the study...).

\subsection{Practical implications}


The results of this research confirm that the level of rumination on COVID-19 is medium-high among students of post-compulsory education, which depends, in part, on the perception of vulnerability to the disease. Given that these circumstances could have a negative effect on attention, concentration, motivation, and, in short, on academic performance, it would be advisable to introduce systems that counteract the effect of rumination on cognitive performance. In this sense, Yamada \& Victor [22] have confirmed that mindfulness practices counteract the negative effects of rumination and have a positive effect on well-being and the capacity for learning in university students. On the other hand, employing a playful job design would be helpful in dealing with ruminant thoughts about COVID-19. This has been demonstrated in the workplace by Bakker \& van Wingerden [16].

\section{Conclusions}

Our findings are consistent with the current understanding of the role of perception of vulnerability to illness and dysfunctional thoughts. Adopting organizational measures that offer security to students against the possibility of being infected by COVID-19, as well as appropriate teaching strategies and methods, could avoid an excess of ruminant thoughts and, consequently, less interference in attention.

Author Contributions: All co-authors contributed to the completion of this paper. Specifically: Conceptualization, J.M.F., C.G.A. and G.T .; Research, J.M.F .; Methodology, J.M.F., C.G.A. and G.T .; Formal Analysis, J.M.F., C.G.A. and G.T .; Writing - Original Draft Preparation, J.M.F., C.G.A. and G.T .; Writing - Review and Editing, J.M.F., C.G.A., G.T; Supervision,, C.G.A. and G.T. All authors have read and agreed to the published version of the manuscript

Funding: This research received no external funding.

Acknowledgments: We appreciate the cooperation of vocational training students in social integration of the IES Alhadra (Almería, Spain) to collect the sample.

Conflicts of Interest: The authors declare no conflict of interest.

\section{References}

1. Polizzi, C., Lynn, S. J.; Perry, A. Stress and coping in the time of COVID-19: Pathways to resilience and recovery. Clinical Neuropsychiatry, 2020, 17, 59 - 62. http://dx.doi.org/10.36131/CN20200204

2. Luhmann, M.; Hofmann, W.; Eid, M.; Lucas, R. E. Subjective well-being and adaptation to life events: A meta-analysis. J Pers Soc Psychol, 2012, 102, 592-615. http://dx.doi.org/10 .1037/a0025948

3. Nolen-Hoeksema, S.; Wisco, B. E.; Lyubomirsky, S. Rethinking rumination. Perspect Psychol Sci, 2008, $3,400-424$. http://dx.doi.org/10.1111/j.1745-6924.2008.00088.x

4. Whitmer, A. J.; Gotlib, I. H. An attentional scope model of rumination. Psychol Bull, 2013, 139(5), 1036-1061. https://doiorg.ezproxy.uned.es/10.1037/a0030923

5. Joormann, J. Cognitive inhibition and emotional regulation. Current Directions in Psychological Science, 2010, 19, 161-166. https://doi:10.1177/ 0963721410370293

6. Watkins, E.; Brown, R. G. Rumination and executive function in depression: An experimental study. J Neurol Neurosurg Psychiatry, 2002, 72, 400 - 402. doi:10.1136/jnnp.72.3.400

7. Koch, I.; Gade, M.; Schuch, S.; Philipp, A. The role of inhibition in task switching: A review. Psychon Bull Rev, 2010, 17, 1-14.

8. Daches, S.; Mor, N.; Winquist, J.; Gilboa-Schectman, E. Brooding and attentional control in processing self-encoded information: Evidence from a modified Garner task. Cognition \& Emotion, 2010, 24, 876 - 885. doi:10.1080/02699930902954825

9. Ahorsu, D. K.; Lin, C.-Y.; Imani, V., Saffari, M.; Griffiths, M. D.; Pakpour, A. H. The fear of covid-19 scale: Development and initial validation. Int J Ment Health Addict, 2020, 1-9. Advance online publication https://doiorg.ezproxy.uned.es/10.1007/s11469-020-00270-8

10. Magallares, A.; Fuster-Ruiz De Apodaca., MJ.; Morales, JF. Psychometric properties and criterion validity of the Perceived Vulnerability to Disease Scale (PVD) in the Spanish population / Propiedades psicométricas y validez de criterio de la escala de Percepción de Vulnerabilidad a la Enfermedad (PVE en población española), Revista de Psicología Social, 2017, 32:1, 164-195, DOI: 10.1080/02134748.2016.1248025

11. Tybur, J. M.; Lieberman, D.; Griskevicius, V. Microbes, mating, and morality: Individual differences in three functional domains of disgust. J Pers Soc Psychol, 2009, 97, 103-122. doi:10.1037/a0015474 
12. Fuster-Ruiz de Apodaca, M. J.; Molero, F.; Gil de Montes, L.; Agirrezabal, A.; Toledo, J.; Jaen, A.; Spanish Group for the Study of HIV-Related Stigma (2014). Evolution of HIV-related stigma in Spain between 2008 and 2012. AIDS care, 2014, 26, Suppl 1, S41-S45. https://doi.org/10.1080/09540121.2014.906557

13. Lyubomirsky, S.; Tkach, C. The consequences of dysphoric rumination. In Rumination: Nature, theory, and treatment of negative thinking in depression; Papageorgiou, C., Wells, A., Eds.; John Wiley \& Sons: Chichester, England, 2003, pp. 21-41. http://dx.doi.org/10.1002/ 9780470713853.ch2

14. Brosschot, J. F.; Gerin, W.; Thayer, J. F. The perseverative cognition hypothesis: A review of worry, prolonged stress-related physiological activation, and health. J Psychosom Res, 2006, 60, 113-124. http://dx.doi.org/10.1016/j.jpsychores.2005.06.074

15. Constantin, K.; English, M. M.; Mazmanian, D. Anxiety, depression, and procrastination among students: Rumination plays a larger mediating role than worry. Journal of Rational-Emotive $\mathcal{E}$ Cognitive-Behavior Therapy, 2018, 36(1), 15-27. https://doiorg.ezproxy.uned.es/10.1007/s10942-017-0271-5

16. Bakker, A. B.; van Wingerden, J. Rumination About COVID-19 and Employee Well-Being: The Role of Playful Work Design. Canadian Psychology/Psychologie Canadienne, Advance online publication, 2020, No Pagination Specified. http://dx.doi.org/10.1037/cap0000262

17. Smith, J. M.; Alloy, L. B. A roadmap to rumination: A review of the definition, assessment, and conceptualization of this multifaceted construct. Clin Psychol Rev, 2009, 29, 116 -128. http://dx.doi.org/ 10.1016/j.cpr.2008.10.003

18. Fernández-Marcos, T. The interference of rumination on attention. Unpublished doctoral thesis, Autonomous University of Madrid [UAM]. Institutional repository- UAM, 2018.

19. Lyubomirsky, S.; Kasri, F.; Zehm, K. Dysphoric Rumination Impairs Concentration on Academic Tasks. Cognitive Therapy and Research, 2003, 27(3), 309-330. https://doi-org.ezproxy.uned.es/10.1023/A:1023918517378

20. Garnefski, N.; Kraaij, V. Specificity of relations between adolescents' cognitive emotion regulation strategies and symptoms of depression and anxiety. Cognition and Emotion, 2018, 32, 1401-1408. http://dx .doi.org/10.1080/02699931.2016.1232698

21. Duncan, L.; Schaller, M.; Park, J. Perceived vulnerability to disease: Development and validation of a 15-item self-report instrument. Personality and Individual Differences, 2009, 47, 541-546. doi:10.1016/j.paid.2009.05.001

22. Yamada, K.; Victor, T. L. The impact of mindful awareness practices on college student health, well-being, and capacity for learning: A pilot study. Psychology Learning $\& \quad$ Teaching, 2012, 11(2), 139-145. https://doiorg.ezproxy.uned.es/10.2304/plat.2012.11.2.139 